\title{
Evaluation of the Rapid Polymyxin NP test and its industrial version for the detection of polymyxin-resistant Enterobacteriaceae
}

\author{
Aurélie Jayol $^{\text {a,b,c }}$, Nicolas Kieffer a,b,c ${ }^{\text {, Laurent Poirel }}{ }^{\text {a,b,c,* }}$, François Guérin ${ }^{\text {d,e }}$, Deniz Güneser ${ }^{\text {a,b }}$, \\ Vincent Cattoir ${ }^{\mathrm{f}}$, Patrice Nordmann ${ }^{\mathrm{a}, \mathrm{b}, \mathrm{c}, \mathrm{g}}$
}

a Emerging Antibiotic Resistance Unit, Medical and Molecular Microbiology, Department of Medicine, Universi
b French INSERM European Unit, University of Fribourg (LEA-IAME), Fribourg, Switzerland
c National Reference Center for Emerging Antibiotic Resistance, Fribourg, Switzerland
d Université de Caen Normandie, EA4655 U2RM (Equipe 'Antibio-résistance'), Caen, France
e CHU de Caen, Service de Microbiologie, Caen, France
${ }^{\mathrm{f}}$ Laboratoire de Bactériologie, CHU de Rennes, Rennes, France
g University of Lausanne and University Hospital Center, Lausanne, Switzerland
* Corresponding author. Tel.: +41-26-300-9582. E-mail address: laurent.poirel@unifr.ch (L. Poirel).

The commercial Rapid Polymyxin NP test was evaluated to detect colistin-resistant Enterobacteriaceae. A total of 223 enterobacterial isolates corresponding to 136 resistant (including 38 MCR-like producers), 19 heteroresistant, and 78 colistin-susceptible isolates were tested. The test was performed according to the manufacturer's instruction, and the color of the wells was read after 2 and 3 hours of incubation. The results were compared with those of the homemade Rapid Polymyxin NP test, and manual broth microdilution according to EUCAST guidelines was used as the reference method to determine the performance of the test. Excellent performance of the commercial Rapid Polymyxin NP test was found with a very major error rate, a major error rate, a sensitivity, and a specificity of $1.9 \%, 5.1 \%, 98.1 \%$, and $94.9 \%$, respectively. The performance of the homemade Polymyxin NP test was similar, with a slightly better value for the very major error (1.2\%).

Polymyxins represent one of the few remaining treatment options for treating carbapenemase-producing Enterobacteriaceae. The increasing use of polymyxins is now associated with increasing isolation of colistin-resistant isolates worldwide (Poirel et al., 2017). Since the identification of the plasmid-mediated colistin resistance gene $m c r-1$, and then more recently of other MCR-like encoding genes, controlling the dissemination of colistin resistance has now become a major concern (Poirel et al., 2017). Indeed, the fear is to isolate carbapenem- and polymyxin-resistant isolates, therefore exhibiting pandrug resistance. It becomes now mandatory to possess reliable and rapid diagnostic techniques for determining polymyxin susceptibility.

The broth microdilution (BMD) technique that is time-consuming $(18 \mathrm{~h}$ ) has been retained as the reference method for evaluating polymyxin susceptibility. A rapid test (Rapid Polymyxin NP test) has been recently developed to detect colistin resistance in Enterobacteriaceae within 2 hours (Nordmann et al., 2016a,b). This test is based on the detection of the glucose metabolism of Enterobacteriaceae upon culture and differentiates colistin-susceptible from colistin-resistant isolates by supplementing the growth medium with a given concentration of colistin. An industrial version of this test is now available (ELITechGroup,
Puteaux, France). The objective of this study was to evaluate the performance of this industrial Rapid Polymyxin NP test to detect colistin resistance and heteroresistance in Enterobacteriaceae.

A collection of 233 enterobacterial isolates was tested using the homemade and the industrial Rapid Polymyxin NP test. It included 136 colistin-resistant, 19 colistin-heteroresistant (Guerin et al., 2016; Hjort et al., 2016), and 78 colistin-susceptible isolates, belonging to various enterobacterial species. Among the 136 colistin-resistant isolates, 10 belonged to bacterial species intrinsically resistant to colistin, 120 had been characterized for their acquired mechanisms of resistance (chromosomally or plasmid encoded), and 6 isolates presented an unknown mechanism of resistance (Table 1). Overall, resistance mechanisms corresponded to substitutions or truncations in the PmrA/PmrB proteins, the $\mathrm{PhoP} / \mathrm{PhoQ}$ regulatory proteins, and the MgrB protein. In addition, 38 of the resistant isolates produced the plasmid-mediated MCR-1, MCR-2, MCR-3, or MCR-4 determinants.

The homemade and industrial Polymyxin NP tests were both performed by using a 3-3.5 McFarland bacterial suspension into $0.9 \%$ $\mathrm{NaCl}$ as recommended (Nordmann et al., 2016a,b, and manufacturer guidelines). Results were interpreted by 2 independent readers after 2 and 3 hours of incubation at $37^{\circ} \mathrm{C}$. A positive test corresponded to an orange-to-yellow color change, whereas an orange color corresponded to a negative test. 
Table 1

Features of the tested strain collection and comparative results between the homemade and commercialized Rapid Polymyxin NP tests.

\begin{tabular}{|c|c|c|c|c|c|c|}
\hline Strains & Species & $\begin{array}{l}\text { Homemade Rapid } \\
\text { Polymyxin } \mathrm{NP}^{\mathrm{a}}\end{array}$ & $\begin{array}{l}\text { Industrial Rapid } \\
\text { Polymyxin } \mathrm{NP}^{\mathrm{a}}\end{array}$ & $\begin{array}{l}\text { MIC of colistin } \\
(\mu \mathrm{g} / \mathrm{mL})\end{array}$ & $\begin{array}{l}\text { Resistance } \\
\text { mechanisms }\end{array}$ & Reference \\
\hline \multicolumn{7}{|c|}{ Resistant isolates } \\
\hline R1583 & P. vulgaris & + & + & $>16$ & Intrinsic & This study \\
\hline R2260 & P. mirabilis & + & + & $>16$ & Intrinsic & This study \\
\hline $\mathrm{R} 2556$ & P. mirabilis & + & + & $>16$ & Intrinsic & This study \\
\hline R1473 & M. morganii & + & + & $>16$ & Intrinsic & This study \\
\hline R1474 & M. morganii & + & + & $>16$ & Intrinsic & This study \\
\hline R1607 & P. stuartii & + & + & $>16$ & Intrinsic & This study \\
\hline R2593 & P. rettgeri & + & + & $>16$ & Intrinsic & This study \\
\hline R2594 & P. rettgeri & + & + & $>16$ & Intrinsic & This study \\
\hline $\mathrm{R} 1278$ & S. marcescens & + & + & $>16$ & Intrinsic & This study \\
\hline $\mathrm{R} 2480$ & S. marcescens & + & + & $>16$ & Intrinsic & This study \\
\hline FR-06 & K. pneumoniae & + & + & 32 & PmrA G53C & (Nordmann et al., 2016a) \\
\hline FR-07 & K. pneumoniae & + & + & 32 & PmrA G53S & (Nordmann et al., 2016a) \\
\hline FR-08 & K. pneumoniae & + & + & 128 & PmrA G53S & (Nordmann et al., 2016a) \\
\hline FR-09 & K. pneumoniae & + & + & 32 & PmrB L17Q & (Nordmann et al., 2016a) \\
\hline FR-10 & K. pneumoniae & + & + & 16 & PmrB T157P & (Nordmann et al., 2016a) \\
\hline FR-11 & K. pneumoniae & + & + & 32 & PmrB T157P & (Nordmann et al., 2016a) \\
\hline FR-12 & K. pneumoniae & + & + & 16 & PmrB T157P & (Nordmann et al., 2016a) \\
\hline FR-13 & K. pneumoniae & + & + & 32 & PmrB T157P & (Nordmann et al., 2016a) \\
\hline FR-14 & K. pneumoniae & + & + & 32 & PmrB T157P & (Nordmann et al., 2016a) \\
\hline FR-15 & K. pneumoniae & + & + & 16 & PmrB T157P & (Nordmann et al., 2016a) \\
\hline FR-16 & K. pneumoniae & + & + & 128 & PhoP D191Y and deletion of $25 \mathrm{nt}$ & (Nordmann et al., 2016a) \\
\hline FR-17 & K. pneumoniae & + & + & $>128$ & PhoQ R16C & (Nordmann et al., 2016a) \\
\hline FR-18 & K. pneumoniae & + & + & 32 & MgrB W20R & (Nordmann et al., 2016a) \\
\hline FR-19 & K. pneumoniae & + & + & 32 & MgrB M27K & (Nordmann et al., 2016a) \\
\hline FR-20 & K. pneumoniae & + & + & 64 & MgrB C39Y & (Nordmann et al., 2016a) \\
\hline FR-22 & K. pneumoniae & + & + & 64 & MgrB I45T & (Nordmann et al., 2016a) \\
\hline FR-23 & K. pneumoniae & + & + & 64 & MgrB P46S & (Nordmann et al., 2016a) \\
\hline FR-24 & K. pneumoniae & + & + & 4 & MgrB W47R & (Nordmann et al., 2016a) \\
\hline FR-25 & K. pneumoniae & + & + & 128 & MgrB truncated (2 aa) & (Nordmann et al., 2016a) \\
\hline FR-26 & K. pneumoniae & + & + & 128 & MgrB truncated (2 aa) & (Nordmann et al., 2016a) \\
\hline FR-27 & K. pneumoniae & + & + & 64 & MgrB truncated (2 aa) & (Nordmann et al., 2016a) \\
\hline FR-28 & K. pneumoniae & + & + & 128 & MgrB truncated (27 aa) & (Nordmann et al., 2016a) \\
\hline FR-31 & K. pneumoniae & + & + & 64 & MgrB truncated (29 aa) & (Nordmann et al., 2016a) \\
\hline FR-32 & K. pneumoniae & + & + & 64 & MgrB truncated (29 aa) & (Nordmann et al., 2016a) \\
\hline FR-33 & K. pneumoniae & + & + & 32 & MgrB truncated (29 aa) & (Nordmann et al., 2016a) \\
\hline FR-34 & K. pneumoniae & + & + & 64 & MgrB truncated (29 aa) & (Nordmann et al., 2016a) \\
\hline FR-35 & K. pneumoniae & + & + & 128 & MgrB truncated (29 aa) & (Nordmann et al., 2016a) \\
\hline FR-36 & K. pneumoniae & + & + & 128 & MgrB truncated (29 aa) & (Nordmann et al., 2016a) \\
\hline FR-37 & K. pneumoniae & + & + & 32 & MgrB truncated (32 aa) & (Nordmann et al., 2016a) \\
\hline FR-38 & K. pneumoniae & + & + & 32 & MgrB truncated (46 aa) & (Nordmann et al., 2016a) \\
\hline FR-39 & K. pneumoniae & + & + & 64 & $m g r B$ truncated by IS1R & (Nordmann et al., 2016a) \\
\hline FR-40 & K. pneumoniae & + & + & 64 & mgrB truncated by ISEcp $1 /$ bla $a_{\mathrm{CTX}-\mathrm{M}-15}$ & (Nordmann et al., 2016a) \\
\hline FR-41 & K. pneumoniae & + & + & 32 & mgrB truncated by IS102-like & (Nordmann et al., 2016a) \\
\hline FR-42 & K. pneumoniae & + & + & $>128$ & mgrB truncated by IS102-like & (Nordmann et al., 2016a) \\
\hline FR-43 & K. pneumoniae & + & + & 64 & $m g r B$ truncated by IS903b & (Nordmann et al., 2016a) \\
\hline FR-44 & K. pneumoniae & + & + & 64 & mgrB truncated by IS2 & (Nordmann et al., 2016a) \\
\hline FR-45 & K. pneumoniae & + & + & 128 & $m g r B$ truncated by IS1R & (Nordmann et al., 2016a) \\
\hline FR-46 & K. pneumoniae & + & + & 64 & mgrB truncated by IS1R & (Nordmann et al., 2016a) \\
\hline FR-47 & K. pneumoniae & + & + & 64 & mgrB truncated by IS903b-like & (Nordmann et al., 2016a) \\
\hline FR-48 & K. pneumoniae & + & + & 128 & mgrB truncated by IS903-like & (Nordmann et al., 2016a) \\
\hline FR-49 & K. pneumoniae & + & + & 64 & mgrB truncated by IS5-like & (Nordmann et al., 2016a) \\
\hline FR-50 & K. pneumoniae & + & + & 16 & mgrB truncated by IS5-like & (Nordmann et al., 2016a) \\
\hline FR-51 & K. pneumoniae & + & + & 64 & mgrB truncated by IS5-like & (Nordmann et al., 2016a) \\
\hline FR-52 & K. pneumoniae & + & + & 128 & mgrB truncated by IS5-like & (Nordmann et al., 2016a) \\
\hline FR-54 & K. pneumoniae & + & + & 128 & mgrB truncated by ISKpn13 & (Nordmann et al., 2016a) \\
\hline FR-56 & K. pneumoniae & + & + & 64 & mgrB truncated by ISKpn26-like & (Nordmann et al., 2016a) \\
\hline FR-58 & K. pneumoniae & + & + & 32 & mgrB truncated by ISKpn26-like & (Nordmann et al., 2016a) \\
\hline FR-62 & K. pneumoniae & + & + & 64 & $m g r B$ truncated by IS903b & (Nordmann et al., 2016a) \\
\hline FR-63 & K. pneumoniae & + & + & 128 & mgrB truncated by IS1R-like & (Nordmann et al., 2016a) \\
\hline FR-67 & K. pneumoniae & + & + & 128 & mgrB truncated by ISKpn26-like & (Nordmann et al., 2016a) \\
\hline FR-68 & K. pneumoniae & + & + & 64 & mgrB truncated by ISKpn14 & (Nordmann et al., 2016a) \\
\hline FR-69 & K. pneumoniae & + & + & 128 & mgrB truncated by IS1R & (Nordmann et al., 2016a) \\
\hline FR-81 & K. pneumoniae & + & + & 64 & Full mgrB gene deletion & (Nordmann et al., 2016a) \\
\hline FR-84 & K. pneumoniae & + & + & 128 & Deletion nt $70 \mathrm{mgrB}$ and substitution nt 73 & (Nordmann et al., 2016a) \\
\hline FR-89 & K. pneumoniae & + & + & $>128$ & Deletion nt 23 to $33 \mathrm{mgrB}$ & (Nordmann et al., 2016a) \\
\hline FR-90 & K. pneumoniae & + & + & 64 & Deletion nt 30 et $31 \mathrm{mgrB}$ & (Nordmann et al., 2016a) \\
\hline FR-87 & K. pneumoniae & + & + & 16 & Deletion nt $100 \mathrm{mgrB}$ & (Nordmann et al., 2016a) \\
\hline FR-30 & K. pneumoniae & + & + & $>128$ & MgrB truncated (27 aa) & (Nordmann et al., 2016a) \\
\hline FR-53 & K. pneumoniae & + & + & 64 & mgrB truncated by IS5-like & (Nordmann et al., 2016a) \\
\hline FR-55 & K. pneumoniae & + & + & $>128$ & mgrB truncated by ISKpn26-like & (Nordmann et al., 2016a) \\
\hline FR-57 & K. pneumoniae & + & + & 128 & mgrB truncated by ISKpn26-like & (Nordmann et al., 2016a) \\
\hline FR-59 & K. pneumoniae & + & + & 32 & $m g r B$ truncated by IS903B & (Nordmann et al., 2016a) \\
\hline
\end{tabular}


Table 1 (continued)

\begin{tabular}{|c|c|c|c|c|c|c|}
\hline Strains & Species & $\begin{array}{l}\text { Homemade Rapid } \\
\text { Polymyxin } \mathrm{NP}^{\mathrm{a}}\end{array}$ & $\begin{array}{l}\text { Industrial Rapid } \\
\text { Polymyxin } \mathrm{NP}^{\mathrm{a}}\end{array}$ & $\begin{array}{l}\text { MIC of colistin } \\
(\mu \mathrm{g} / \mathrm{mL})\end{array}$ & $\begin{array}{l}\text { Resistance } \\
\text { mechanisms }\end{array}$ & Reference \\
\hline FR-60 & K. pneumoniae & + & + & 128 & mgrB ISKpn14 between +77 and +78 & (Nordmann et al., 2016a) \\
\hline FR-61 & K. pneumoniae & + & + & 128 & mgrB duplication $19 \mathrm{nt}$ between +84 and +85 & (Nordmann et al., 2016a) \\
\hline FR-64 & K. pneumoniae & + & + & 64 & $m g r B$ truncated by IS903b-like & (Nordmann et al., 2016a) \\
\hline FR-65 & K. pneumoniae & + & + & 8 & mgrB truncated by IS1R & (Nordmann et al., 2016a) \\
\hline FR-66 & K. pneumoniae & + & + & 32 & mgrB truncated by IS1R & (Nordmann et al., 2016a) \\
\hline FR-70 & K. pneumoniae & + & + & 128 & mgrB truncated by IS10R & (Nordmann et al., 2016a) \\
\hline FR-71 & K. pneumoniae & + & + & 64 & mgrB truncated by ISKpn14 & (Nordmann et al., 2016a) \\
\hline FR-72 & K. pneumoniae & + & + & 64 & mgrB truncated by ISKpn14 & (Nordmann et al., 2016a) \\
\hline FR-73 & K. pneumoniae & + & + & 64 & mgrB truncated by IS1R & (Nordmann et al., 2016a) \\
\hline FR-74 & K. pneumoniae & + & + & 128 & $m g r B$ truncated by IS1R & (Nordmann et al., 2016a) \\
\hline FR-75 & K. pneumoniae & + & + & 64 & mgrB truncated by IS $1 R$ & (Nordmann et al., 2016a) \\
\hline FR-76 & K. pneumoniae & + & + & 32 & $m g r B$ truncated by IS1R & (Nordmann et al., 2016a) \\
\hline FR-77 & K. pneumoniae & + & + & 32 & mgrB truncated by ISKpn14-like & (Nordmann et al., 2016a) \\
\hline FR-78 & K. pneumoniae & + & + & 32 & mgrB truncated by IS1R & (Nordmann et al., 2016a) \\
\hline FR-79 & K. pneumoniae & + & + & 16 & Full $m g r B$ gene deletion & (Nordmann et al., 2016a) \\
\hline FR-80 & K. pneumoniae & + & + & $>128$ & Full $m g r B$ gene deletion & (Nordmann et al., 2016a) \\
\hline FR-82 & K. pneumoniae & + & + & 64 & Full $m g r B$ gene deletion & (Nordmann et al., 2016a) \\
\hline FR-83 & K. pneumoniae & + & + & 32 & Deletion nt 23 mgrB & (Nordmann et al., 2016a) \\
\hline FR-85 & K. pneumoniae & + & + & 64 & Deletion nt 74 mgrB & (Nordmann et al., 2016a) \\
\hline FR-86 & K. pneumoniae & + & + & 64 & Deletion nt $100 \mathrm{mgrB}$ & (Nordmann et al., 2016a) \\
\hline FR-93 & E. coli & + & + & 4 & Plasmid-mediated $\mathrm{mcr}$ - 1 gene & (Nordmann et al., 2016a) \\
\hline FR-94 & E. coli & + & + & 16 & Plasmid-mediated $m c r-1$ gene & (Nordmann et al., 2016a) \\
\hline FR-95 & E. coli & + & + & 16 & Plasmid-mediated $\mathrm{mcr}$-1 gene & (Nordmann et al., 2016a) \\
\hline FR-96 & E. coli & + & + & 16 & Plasmid-mediated $\mathrm{mcr}$-1 gene & (Nordmann et al., 2016a) \\
\hline FR-97 & E. coli & + & + & 16 & Plasmid-mediated $m c r-1$ gene & (Nordmann et al., 2016a) \\
\hline FR-98 & E. coli & + & + & 8 & Plasmid-mediated $\mathrm{mcr}-1$ gene & (Nordmann et al., 2016a) \\
\hline FR-99 & E. coli & + & + & 16 & Plasmid-mediated $\mathrm{mcr}-1$ gene & (Nordmann et al., 2016a) \\
\hline Af 48 & E. coli & + & + & 8 & Plasmid-mediated $m c r-1$ gene & Poirel et al., 2016 \\
\hline CDF1 & E. coli & + & + & 16 & Plasmid-mediated $m c r-1$ gene & (Nordmann et al., 2016a) \\
\hline CDF2 & E. coli & + & + & 16 & Plasmid-mediated $m c r-1$ gene & (Nordmann et al., 2016a) \\
\hline CDF6 & E. coli & + & + & 16 & Plasmid-mediated $\mathrm{mcr}$ - 1 gene & (Kieffer et al., 2017b) \\
\hline CDF8 & E. coli & + & + & 8 & Plasmid-mediated $\mathrm{mcr}-1$ gene & (Kieffer et al., 2017b) \\
\hline AGU & E. coli & + & + & 8 & Plasmid-mediated $m c r-1$ gene & This study \\
\hline DJE & E. coli & + & + & 8 & Plasmid-mediated $\mathrm{mcr}-1$ gene & This study \\
\hline S115 & E. coli & + & + & 16 & Plasmid-mediated $m c r-1$ gene & (Nordmann et al., 2016b) \\
\hline $\mathrm{P} 4$ & E. coli & + & + & 8 & Plasmid-mediated $m c r-1$ gene & (Kieffer et al., 2017a) \\
\hline P26 & E. coli & + & + & 8 & Plasmid-mediated $m c r-1$ gene & (Kieffer et al., 2017a) \\
\hline B4 & E. coli & + & + & 4 & Plasmid-mediated $m c r-1$ gene & (Kieffer et al., 2017a) \\
\hline B8 & E. coli & + & + & 8 & Plasmid-mediated $\mathrm{mcr}$-1 gene & (Kieffer et al., 2017a) \\
\hline B9 & E. coli & + & + & 16 & Plasmid-mediated $m c r-1$ gene & (Kieffer et al., 2017a) \\
\hline $\mathrm{B} 12$ & E. coli & + & + & 8 & Plasmid-mediated $m c r-1$ gene & (Kieffer et al., 2017a) \\
\hline $\mathrm{B} 15$ & E. coli & + & + & 8 & Plasmid-mediated $m c r-1$ gene & (Kieffer et al., 2017a) \\
\hline B18 & E. coli & + & + & 8 & Plasmid-mediated $m c r-1$ gene & (Kieffer et al., 2017a) \\
\hline B19 & E. coli & + & + & 16 & Plasmid-mediated $m c r-1$ gene & (Kieffer et al., 2017a) \\
\hline $\mathrm{B} 20$ & E. coli & + & + & 8 & Plasmid-mediated $m c r-1$ gene & (Kieffer et al., 2017a) \\
\hline B22 & E. coli & + & + & 8 & Plasmid-mediated $\mathrm{mcr}$-1 gene & (Kieffer et al., 2017a) \\
\hline B47 & E. coli & + & + & 8 & Plasmid-mediated $\mathrm{mcr}-1$ gene & (Kieffer et al., 2017a) \\
\hline B50 & E. coli & + & + & 8 & Plasmid-mediated $\mathrm{mcr}$-1 gene & (Kieffer et al., 2017a) \\
\hline C2729 & E. coli & + & + & 8 & Plasmid-mediated $m c r-1$ gene & (Kieffer et al., 2017a) \\
\hline C2730 & E. coli & + & + & 8 & Plasmid-mediated $m c r-1$ gene & (Kieffer et al., 2017a) \\
\hline B31K & K. pneumoniae & + & + & 16 & Plasmid-mediated $\mathrm{mcr}-1$ gene & This study \\
\hline P26 & K. pneumoniae & + & + & 8 & Plasmid-mediated $m c r-1$ gene & This study \\
\hline C2728 & K. pneumoniae & + & + & 16 & Plasmid-mediated $m c r-1$ gene & This study \\
\hline C2731 & S. enterica & + & + & 8 & Plasmid-mediated $m c r-1$ gene & This study \\
\hline C2732 & S. enterica & + & + & 16 & Plasmid-mediated $\mathrm{mcr}-1$ gene & This study \\
\hline R2812 & E. coli & + & + & 4 & Plasmid-mediated mor-2 gene & (Xavier et al., 2016) \\
\hline R3181 & E. coli & + & + & 4 & Plasmid-mediated mcr-3-like gene & This study \\
\hline S136 & S. enterica & + & + & 4 & Plasmid-mediated mor-4-like gene & This study \\
\hline FR-119 & E. coli & - & - & 8 & Unknown & This study \\
\hline FR-109 & K. pneumoniae & + & + & 64 & Unknown & This study \\
\hline FR-115 & K. pneumoniae & + & + & 64 & Unknown & This study \\
\hline FR-103 & K. pneumoniae & + & + & 4 & Unknown & This study \\
\hline FR-118 & K. pneumoniae & + & + & 4 & Unknown & This study \\
\hline FR-121 & E. coli & + & + & 4 & Unknown & This study \\
\hline \multicolumn{7}{|c|}{ Heteroresistant isolates } \\
\hline DA27007 & S. enterica & + & + & 4 & Chromosomal amplification of pmrD gene & (Hjort et al., 2016) \\
\hline DA33039 & S. enterica & + & + & 4 & Chromosomal amplification of $p m r D$ gene & (Hjort et al., 2016) \\
\hline R2914 & E. cloacae & + & + & $>256$ & Cluster XI & (Guerin et al., 2016) \\
\hline R2915 & E. cloacae & + & + & $>256$ & Cluster I & (Guerin et al., 2016) \\
\hline R2917 & E. cloacae & + & $+(3 h)$ & $>256$ & Cluster I & (Guerin et al., 2016) \\
\hline R2919 & E. cloacae & + & + & 256 & Cluster II & (Guerin et al., 2016) \\
\hline R2922 & E. cloacae & + & - & 16 & Cluster IV & (Guerin et al., 2016) \\
\hline R2923 & E. cloacae & - & - & 16 & Cluster IV & (Guerin et al., 2016) \\
\hline R2928 & E. cloacae & + & + & 64 & Cluster VII & (Guerin et al., 2016) \\
\hline R2929 & E. cloacae & + & + & 256 & Cluster VII & (Guerin et al., 2016) \\
\hline
\end{tabular}


Table 1 (continued)

\begin{tabular}{|c|c|c|c|c|c|c|}
\hline Strains & Species & $\begin{array}{l}\text { Homemade Rapid } \\
\text { Polymyxin } \mathrm{NP}^{\mathrm{a}}\end{array}$ & $\begin{array}{l}\text { Industrial Rapid } \\
\text { Polymyxin } \mathrm{NP}^{\mathrm{a}}\end{array}$ & $\begin{array}{l}\text { MIC of colistin } \\
(\mu \mathrm{g} / \mathrm{mL})\end{array}$ & $\begin{array}{l}\text { Resistance } \\
\text { mechanisms }\end{array}$ & Reference \\
\hline R2932 & E. cloacae & + & $+(3 \mathrm{~h})$ & 256 & Cluster IX & (Guerin et al., 2016) \\
\hline R2933 & E. cloacae & + & $+(3 \mathrm{~h})$ & 64 & Cluster IX & (Guerin et al., 2016) \\
\hline R2936 & E. cloacae & + & + & 128 & Cluster XI & (Guerin et al., 2016) \\
\hline R2938 & E. cloacae & + & + & $>256$ & Cluster XII & (Guerin et al., 2016) \\
\hline R2939 & E. cloacae & + & + & 128 & Cluster XII & (Guerin et al., 2016) \\
\hline R2945 & E. cloacae & + & + & 256 & Cluster XI & (Guerin et al., 2016) \\
\hline R2946 & E. cloacae & + & + & 16 & Cluster XI & (Guerin et al., 2016) \\
\hline R2947 & E. cloacae & + & + & 256 & Cluster XI & (Guerin et al., 2016) \\
\hline R2950 & E. cloacae & + & + & 16 & Cluster XI & (Guerin et al., 2016) \\
\hline \multicolumn{7}{|c|}{ Susceptible isolates } \\
\hline ATCC 25922 & E. coli & - & - & 0.125 & Wild-type & Reference strain \\
\hline FR-180 & K. pneumoniae & + & + & 1 & NA & (Nordmann et al., 2016a) \\
\hline FR-181 & K. pneumoniae & + & + & 2 & NA & (Nordmann et al., 2016a) \\
\hline FR-182 & K. pneumoniae & + & + & 2 & NA & (Nordmann et al., 2016a) \\
\hline C451 & E. coli & - & - & 1 & NA & This study \\
\hline C889 & K. pneumoniae & - & - & 2 & NA & This study \\
\hline C861 & K. pneumoniae & + & + & 2 & NA & This study \\
\hline FR-165 & K. pneumoniae & - & - & 1 & NA & (Nordmann et al., 2016a) \\
\hline C349 & K. pneumoniae & - & - & $<1$ & NA & This study \\
\hline C352 & E. coli & - & - & $<1$ & NA & This study \\
\hline C353 & E. coli & - & - & $<1$ & NA & This study \\
\hline C355 & E. coli & - & - & $<1$ & NA & This study \\
\hline C358 & E. coli & - & - & $<1$ & NA & This study \\
\hline C360 & E. coli & - & - & $<1$ & NA & This study \\
\hline C361 & E. coli & - & - & $<1$ & NA & This study \\
\hline C468 & E. coli & - & - & $<1$ & NA & This study \\
\hline C469 & E. coli & - & - & $<1$ & NA & This study \\
\hline C470 & C. koseri & - & - & $<1$ & NA & This study \\
\hline C472 & E. aerogenes & - & - & 0.25 & NA & This study \\
\hline C474 & C. koseri & - & - & $<1$ & NA & This study \\
\hline C481 & K. oxytoca & - & - & $<1$ & NA & This study \\
\hline C490 & K. pneumoniae & - & - & $<1$ & NA & This study \\
\hline C508 & K. pneumoniae & - & - & $<1$ & NA & This study \\
\hline C509 & K. pneumoniae & - & - & $<1$ & NA & This study \\
\hline C512 & E. aerogenes & - & - & $<1$ & NA & This study \\
\hline C519 & C. koseri & - & - & $<1$ & NA & This study \\
\hline C524 & K. pneumoniae & - & - & $<1$ & NA & This study \\
\hline C533 & K. pneumoniae & - & - & $<1$ & NA & This study \\
\hline C539 & C. koseri & - & - & $<1$ & NA & This study \\
\hline C548 & E. aerogenes & - & - & $<1$ & NA & This study \\
\hline C554 & K. pneumoniae & - & - & $<1$ & NA & This study \\
\hline C597 & K. pneumoniae & - & - & $<1$ & NA & This study \\
\hline C607 & K. pneumoniae & - & - & $<1$ & NA & This study \\
\hline C632 & E. aerogenes & - & - & $<1$ & NA & This study \\
\hline C635 & C. koseri & - & - & $<1$ & NA & This study \\
\hline C867 & C. koseri & - & - & $<1$ & NA & This study \\
\hline C901 & K. pneumoniae & - & - & 0.5 & NA & This study \\
\hline C914 & K. pneumoniae & - & - & 0.125 & NA & This study \\
\hline C937 & C. koseri & - & - & $<1$ & NA & This study \\
\hline C1029 & K. oxytoca & - & - & $<1$ & NA & This study \\
\hline C1033 & E. cloacae & - & - & $<1$ & NA & This study \\
\hline C1066 & E. coli & - & - & $<1$ & NA & This study \\
\hline C1077 & E. cloacae & - & - & $<1$ & NA & This study \\
\hline C1082 & K. oxytoca & - & - & $<1$ & NA & This study \\
\hline C1085 & C. koseri & - & - & $<1$ & NA & This study \\
\hline C1087 & K. oxytoca & - & - & $<1$ & NA & This study \\
\hline C1216 & E. cloacae & - & - & $<1$ & NA & This study \\
\hline C1221 & E. aerogenes & - & - & $<1$ & NA & This study \\
\hline C1231 & K. oxytoca & - & - & $<1$ & NA & This study \\
\hline C1234 & K. oxytoca & - & - & $<1$ & NA & This study \\
\hline C1285 & E. aerogenes & - & - & $<1$ & NA & This study \\
\hline C1288 & E. aerogenes & - & - & $<1$ & NA & This study \\
\hline C1293 & E. aerogenes & - & - & $<1$ & NA & This study \\
\hline C1397 & K. oxytoca & - & - & $<1$ & NA & This study \\
\hline C1591 & C. freundii & - & - & $<1$ & NA & This study \\
\hline C1637 & E. cloacae & - & - & $<1$ & NA & This study \\
\hline C1664 & K. oxytoca & - & - & $<1$ & NA & This study \\
\hline C1671 & C. freundii & - & - & $<1$ & NA & This study \\
\hline C1703 & E. cloacae & - & - & $<1$ & NA & This study \\
\hline C1753 & E. cloacae & - & - & $<1$ & NA & This study \\
\hline C1877 & C. freundii & - & - & $<1$ & NA & This study \\
\hline C2155 & C. freundii & - & - & $<1$ & NA & This study \\
\hline C2635 & Salmonella spp. & - & - & $<1$ & NA & This study \\
\hline C3690 & C. freundii & - & - & $<1$ & NA & This study \\
\hline
\end{tabular}


Table 1 (continued)

\begin{tabular}{|c|c|c|c|c|c|c|}
\hline Strains & Species & $\begin{array}{l}\text { Homemade Rapid } \\
\text { Polymyxin } \mathrm{NP}^{\mathrm{a}}\end{array}$ & $\begin{array}{l}\text { Industrial Rapid } \\
\text { Polymyxin NPa }\end{array}$ & $\begin{array}{l}\text { MIC of colistin } \\
(\mu \mathrm{g} / \mathrm{mL})\end{array}$ & $\begin{array}{l}\text { Resistance } \\
\text { mechanisms }\end{array}$ & Reference \\
\hline R281 & E. asburiae & - & - & $<1$ & NA & This study \\
\hline R1414 & C. freundii & - & - & $<1$ & NA & This study \\
\hline R1415 & C. freundii & - & - & $<1$ & NA & This study \\
\hline R1416 & C. freundii & - & - & $<1$ & NA & This study \\
\hline R1527 & S. Concord & - & - & $<1$ & NA & This study \\
\hline R1528 & Salmonella spp. & - & - & $<1$ & NA & This study \\
\hline R1529 & S. Isangi & - & - & $<1$ & NA & This study \\
\hline C362 & K. pneumoniae & - & - & 0.125 & NA & This study \\
\hline C367 & K. pneumoniae & - & - & 0.125 & NA & This study \\
\hline C370 & K. pneumoniae & - & - & 0.125 & NA & This study \\
\hline C382 & K. pneumoniae & - & - & 0.125 & NA & This study \\
\hline C383 & K. pneumoniae & - & - & 0.125 & NA & This study \\
\hline C609 & K. pneumoniae & - & - & 0.125 & NA & This study \\
\hline C643 & K. pneumoniae & - & - & 0.125 & NA & This study \\
\hline
\end{tabular}

${ }^{a}$ Isolates with discordant results are shaded.

MICs of colistin were determined in parallel by BMD using cationadjusted Muller Hinton broth (Bio-Rad, Cressier, Switzerland), and Escherichia coli ATCC 25922 was used as control strain. In case of "skip wells" (likely indicating heteroresistance), MICs were determined by considering the well with the highest concentration in which growth was observed, regardless the clear wells (Guerin et al., 2016). Isolates with MICs of colistin $>2 \mu \mathrm{g} / \mathrm{mL}$ were considered as resistant, whereas isolates showing a MIC $\leq 2 \mu \mathrm{g} / \mathrm{mL}$ were considered as susceptible. Results of the 2 Polymyxin NP tests read after 3 hours of incubation were compared with those of the BMD. Errors were ranked as follows: very major error (VME) defined by isolates categorized as susceptible using the marketed panel but resistant by the BMD reference method (false-susceptibility result), and major error (ME) defined by isolates categorized as resistant using the marketed panel but susceptible by the BMD reference method (false-resistant result).

Among the susceptible isolates tested $(n=78), 4 \mathrm{ME}(5.1 \%)$ were detected with both tests giving a specificity of $94.9 \%$ (74/78). Those false-positive results corresponded to isolates having MICs of colistin close to the breakpoint value (i.e., $1-2 \mu \mathrm{g} / \mathrm{mL}$ ).

Among the resistant isolates $(\mathrm{n}=155), 2 \mathrm{VME}(1.2 \%)$ and $3 \mathrm{VME}$ (1.9\%) were detected with both the homemade and of the industrial Rapid Polymyxin NP tests, giving sensitivity rates of 98.7\% (153/155) and $98.1 \%(152 / 155)$, respectively. Experiments were reproduced twice with distinct reading persons and results showed perfect agreement. A single E. coli with an unknown mechanism of resistance was not detected by using both tests. A single and 2 heteroresistant Enterobacter cloacae isolates were not detected with the homemade and of the industrial Rapid Polymyxin NP tests, respectively. Noteworthy, 3 heteroresistant $E$. cloacae isolates were detected after 2 hours of incubation with the homemade test, whereas they were detected after 3 hours of incubation with the industrial test. The performance of the homemade test was therefore quite equivalent to the industrial test for detection of colistin heteroresistance. Such detection is challenging because most of the routine methods (Etest, Vitek-2 automated system) give false-susceptible results for such isolates (Poirel et al., 2017). Recently, an evaluation of the homemade Rapid Polymyxin NP test for detection of colistin heteroresistance in Enterobacter spp. was performed, using a collection of 25 isolates, and a low sensitivity (25\%) was actually found (Simar et al., 2017). Such difference of results remains unexplained, but it is worth highlighting that the strains tested were different. Further studies performed in different laboratories will be needed to evaluate the reliability of the Polymyxin NP tests to detect colistin heteroresistance.

Noteworthy, all the 38 isolates producing a plasmid-mediated colistin resistance determinant (MCR-1 to MCR-4) were perfectly detected by the 2 methods within 2 hours.

To conclude, the homemade and the industrial Polymyxin NP tests showed high sensitivity (98.1\%-98.7\%) and specificity (94.9\%) for detection of colistin resistance in Enterobacteriaceae, regardless of the resistance mechanisms. Those tests therefore possess the potential to be reliable tools for screening colistin-resistant Enterobacteriaceae in clinical laboratories.

\section{Acknowledgments}

This work has been funded by the University of Fribourg and by the Swiss National Reference Center for Emerging Antibiotic Resistance, Fribourg, Switzerland. It has also been funded by the Swiss National Science Foundation (projects FNS-407240_177381 and FNS407240_177382). We thank the following colleagues who provided colistin-resistant isolates: A. Brink, J.W. Decousser, J. FernandezDominguez, J.Y. Madec, M.V. Villegas, M. Yilmaz, D.I. Andersson, and H. Nicoloff.

\section{Conflict of Interest}

None to declare.

\section{References}

Guerin F, Isnard C, Sinel C, Morand P, Dhalluin A, Cattoir V, et al. Cluster-dependent colistin hetero-resistance in Enterobacter cloacae complex. J Antimicrob Chemother 2016. https://doi.org/10.1093/jac/dkw260. [dkw260 [pii]].

Hjort K, Nicoloff $\mathrm{H}$, Andersson DI. Unstable tandem gene amplification generates heteroresistance (variation in resistance within a population) to colistin in Salmonella enterica. Mol Microbiol 2016;102:274-89. https://doi.org/10.1111/mmi.13459.

Kieffer N, Aires-de-Sousa M, Nordmann P, Poirel L. High rate of MCR-1-producing Escherichia coli and Klebsiella pneumoniae among pigs, Portugal. Emerg Infect Dis 2017a;23:2023-9. https://doi.org/10.3201/eid2312.170883.

Kieffer N, Poirel L, Clerc O, Lienhard R, Nordmann P. Co-production of MCR-1 and extended-spectrum $\beta$-lactamase in Escherichia coli recovered from urinary tract infections in Switzerland. Infection 2017b. https://doi.org/10.1007/s15010-017-10806

Nordmann P, Jayol A, Poirel L. Rapid detection of polymyxin resistance in Enterobacteriaceae. Emerg Infect Dis 2016;22:1038-43. https://doi.org/10.3201/eid2206.151840.

Nordmann P, Lienhard R, Kieffer N, Clerc O, Poirel L. Plasmid-mediated colistin-resistant Escherichia coli in bacteremia in Switzerland. Clin Infect Dis 2016;62:1322-3. https://doi.org/10.1093/cid/ciw124. [ciw124 [pii]].

Poirel L, Jayol A, Nordmann P. Polymyxins: antibacterial activity, susceptibility testing and resistance mechanisms encoded by plasmids or chromosomes. Clin Microbiol Rev 2017;30:557-96. https://doi.org/10.1128/CMR.00064-16.

Poirel L, Kieffer N, Brink A, Coetze J, Jayol A, Nordmann P. Genetic features of MCR-1-producing colistin-resistant Escherichia coli isolates, South Africa. Antimicrob Agents Chemother 2016. https://doi.org/10.1128/AAC.00444-16. [AAC.00444-16 [pii]].

Simar S, Sibley D, Ashcraft D, Pankey G. Evaluation of the Rapid Polymyxin NP test for Polymyxin B resistance detection using Enterobacter cloacae and Enterobacter aerogenes isolates. J Clin Microbiol 2017;55:3016-20. https://doi.org/10.1128/JCM. 00934-17.

Xavier BB, Lammens C, Ruhal R, Kumar-Singh S, Butaye P, Goossens H, et al. Identification of a novel plasmid-mediated colistin-resistance gene, $\mathrm{mcr}$-2, in Escherichia coli, Belgium, June 2016. Euro Surveill 2016;21. https://doi.org/10.2807/1560-7917.ES. 2016.21.27.30280. [30280 [pii]]. 